\title{
Voting Rules that are Unbiased but not Transitive-Symmetric
}

\author{
Aadyot Bhatnagar \\ California Institute of Technology \\ Pasadena, CA, U.S.A. \\ aadyotb@gmail.com
}

Submitted: Jun 16, 2019; Accepted: Dec 1, 2019; Published: Jan 10, 2020

(C) The author. Released under the CC BY license (International 4.0).

\begin{abstract}
We explore the relation between two natural symmetry properties of voting rules. The first is transitive-symmetry - the property of invariance to a transitive permutation group - while the second is the "unbiased" property of every voter having the same influence for all i.i.d. probability measures. We show that these properties are distinct by two constructions - one probabilistic, one explicit - of rules that are unbiased but not transitive-symmetric.
\end{abstract}

Mathematics Subject Classifications: 91B12, 05C80

\section{Introduction}

We study voting rules: functions $\Phi:\{-1,1\}^{n} \rightarrow\{-1,1\}$ that map a voting profile the preferences of a set $V=[n]$ of $n$ voters between alternatives -1 and 1 - to an outcome in $\{-1,1\}$. We restrict our attention to voting rules $\Phi$ that are odd, i.e. such that $\Phi(-x)=-\Phi(x)$, and monotone, i.e. such that $\Phi(x) \geqslant \Phi\left(x^{\prime}\right)$ whenever $x \geqslant x^{\prime}$ coordinate-wise.

Let $S_{n}$ be the set of permutations over $V$. For a permutation $\sigma \in S_{n}$, we define a permutation of a voting rule $\Phi$ as $(\sigma \Phi)(x)=\Phi\left(\sigma^{-1}(x)\right)$. We say that $\sigma$ is an automorphism of $\Phi$ if $\sigma \Phi=\Phi$. We denote by aut $(\Phi)$ the automorphism group of $\Phi$. A voting rule $\Phi$ is said to be transitive-symmetric if $\operatorname{aut}(\Phi)$ is transitive, i.e. for every pair of voters $u, v \in V$, there is an automorphism $\sigma \in \operatorname{aut}(\Phi)$ such that $\sigma(u)=v$. Intuitively, transitivesymmetric voting rules define "equitable" elections where each voter plays the same "role" in determining the final outcome. These objects were studied in detail by [2], who call them "equitable voting rules."

For any voting rule $\Phi$ and a probability measure $q$ over $\{-1,1\}^{n}$, voter $i$ 's influence on $\Phi$ is the probability that they are pivotal for a voting profile chosen from $q$. More 
formally, for any $x \in\{-1,1\}^{n}$, let $x^{\oplus i}=\left(x_{1}, \ldots, x_{i-1},-x_{i}, x_{i+1}, \ldots, x_{n}\right)$ be the voting profile obtained when only voter $i$ changes their vote from $x$. We say $i$ is pivotal for $x$ in $\Phi$ if $\Phi(x) \neq \Phi\left(x^{\oplus i}\right)$, and the influence of $i$ on $\Phi$ with respect to probability measure $q$ on $\{-1,1\}^{n}$ is

$$
\mathbb{I}_{i}^{p}(\Phi)=\underset{x \sim q}{\mathbb{P}}\left[\Phi(x) \neq \Phi\left(x^{\oplus i}\right)\right]
$$

We say that a voting rule $\Phi$ is unbiased if $\mathbb{I}_{1}^{q}(\Phi)=\cdots=\mathbb{I}_{n}^{q}(\Phi)$ for every i.i.d. probability measure $q=p^{\otimes n}$ on $\{-1,1\}^{n}$. It is easy to see that all transitive-symmetric voting rules are unbiased, but it is unclear whether the converse holds. Our main theorem shows that the two properties are distinct: ${ }^{1}$

Theorem 1. For all $n$ odd and large enough, there exist odd, monotone voting rules $\Phi:\{-1,1\}^{n} \rightarrow\{-1,1\}$ that are unbiased but not transitive-symmetric.

We prove Theorem 1 by introducing a class of graphic voting rules defined by an underlying graph (Section 2). Informally, we associate transitive-symmetric voting rules to vertex-transitive graphs, and unbiased voting rules to regular graphs. This construction reduces our task to proving the existence of $d$-regular graphs that satisfy certain properties. In Section 3, we provide a probabilistic existence proof inspired by [4] showing that the graphic voting rules of random regular graphs are unbiased and have trivial automorphism groups with probability $1-o(1)$. Section 4 provides an explicit construction of a voting rule that is unbiased but not transitive-symmetric by composing a specific asymmetric graphic voting rule with a transitive-symmetric voting rule. However, this construction is only valid for specific $n$. Section 5 concludes.

\section{Preliminaries}

\subsection{Graph Theory}

Let $G=(V, E)$ be an unweighted, undirected graph with vertex set $V=[n]$ and edge set $E$. We intentionally overload notation because we will associate each vertex of a graph $G$ to a voter in a voting rule $\Phi_{G}$. For any two vertices $u, v \in V$, let $d(u, v)$ denote the length of a shortest path between $u$ and $v$ in $G$. The ball of radius $r$ about a vertex $v$ in $G$ is the set of all points a distance at most $r$ away from $v$, i.e. $B_{r}(v)=\{u \in V$ : $d(u, v) \leqslant r\}$. Analogously, we also let $B_{r}(G)=\left\{B_{r}(v): v \in V\right\}$ denote the set of all balls of radius $r$ in $G$. The diameter of $G$ is $\operatorname{diam}(G)=\max _{u, v \in V} d(u, v)$, and the radius of $G$ is $\operatorname{rad}(G)=\lceil\operatorname{diam}(G) / 2\rceil$. While this definition of the graph radius is non-standard, it has the attractive property that $\operatorname{rad}(G)=\min \left\{r \in \mathbb{R}: B_{r}(u) \cap B_{r}(v) \neq \varnothing \forall u, v \in V\right\}$.

For a permutation $\sigma \in S_{n}$, let $\sigma(G)=(V,\{\{\sigma(u), \sigma(v)\}:\{u, v\} \in E\})$ be the graph obtained by using $\sigma$ to permute the endpoints of every edge in $G$. If $\sigma(G)=G$, then we say that $\sigma$ is an automorphism of $G$, or $\sigma \in \operatorname{aut}(G)$. Analogously, if $B_{r}(\sigma(G))=B_{r}(G)$, then we say that $\sigma$ is an automorphism of $B_{r}(G)$, or $\sigma \in \operatorname{aut}\left(B_{r}(G)\right)$. Note that while

\footnotetext{
${ }^{1}$ We only consider odd $n$ because it is a long-standing open question for which even $n$ there exist odd, monotone, transitive-symmetric voting rules [3].
} 


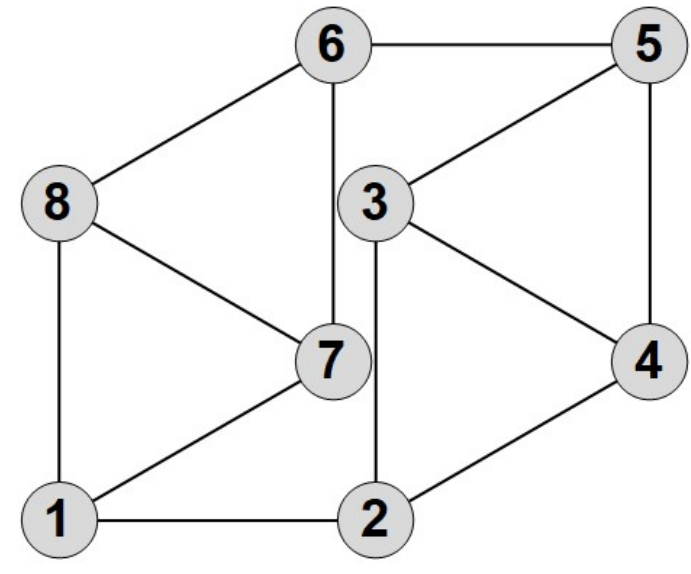

(a) This 3-regular graph is not vertex-transitive, though it still has nontrivial automorphisms.

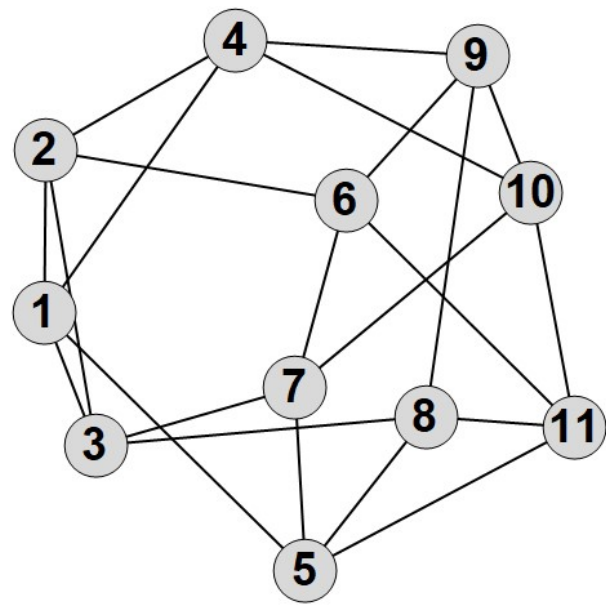

(b) This 4-regular graph is totally asymmetric, i.e. it has no non-trivial automorphisms.

Figure 1: Regular graphs that are not vertex-transitive and have $\operatorname{aut}(G)=\operatorname{aut}\left(B_{1}(G)\right)$. The graphic voting rule associated with the graph in Figure $1 \mathrm{~b}$ is unbiased but not transitive-symmetric.

$\operatorname{aut}(G) \subseteq \operatorname{aut}\left(B_{r}(G)\right)$, it is unclear when the converse holds. Figure 1 provides two examples where $\operatorname{aut}(G)=\operatorname{aut}\left(B_{1}(G)\right)$.

We say that a graph is vertex-transitive if for every pair of vertices $u, v \in V$, there is an automorphism $\sigma \in \operatorname{aut}(G)$ such that $\sigma(u)=v$. We say that $G$ is asymmetric if $|\operatorname{aut}(G)|=1$, i.e. the identity is the only automorphism of $G$. We define vertextransitivity and asymmetry for $B_{r}(G)$ analogously. Finally, we call a graph $d$-regular if all of its vertices have degree $d$. It is easy to see that all vertex-transitive graphs must be regular, though the converse need not hold (Figure 1).

\subsection{Winning Coalitions of Voting Rules}

A winning coalition for a voting rule $\Phi$ is a set of voters $S \subseteq[n]$ such that if a voting profile $x \in\{-1,1\}^{n}$ has $x_{i}=y$ for all $i \in S$, then $\Phi(x)=y$. We say that a winning coalition $S$ is minimal if no strict subset $T \subset S$ of it is also a winning coalition. We note that $\Phi$ is odd and monotone if and only if the winning coalitions for both alternatives -1 and 1 are equal, and any superset of a winning coalition is also a winning coalition. This observation gives rise to the following characterization of odd, monotone voting rules:

Definition 2. For any odd $n$, let $\mathcal{F} \subseteq 2^{[n]}$ be a family of sets such that $T \cap S \neq \varnothing$ and $S \not \subset T$ for all $S, T \in \mathcal{F}$. Define the voting rule $\Phi_{\mathcal{F}}:\{-1,1\}^{n} \rightarrow\{-1,1\}$ as

$$
\Phi_{\mathcal{F}}(x)= \begin{cases}y & \exists S \in \mathcal{F} \text { s.t. } x_{i}=y \forall i \in S \\ \operatorname{maj}_{n}(x) & \text { otherwise }\end{cases}
$$


$\Phi_{\mathcal{F}}$ is well-defined only if $\mathcal{F}$ is an intersecting family of sets. This is because every $S \in \mathcal{F}$ is a minimal winning coalition for $\Phi_{\mathcal{F}}$ (minimality follows because $S \not \subset T$ for any $S, T \in \mathcal{F}$ ). We call a voter $i$ pivotal for a winning coalition $S$ if $S \backslash\{i\}$ is not a winning coalition. This re-framing of voting rules in terms of winning coalitions gives us the following characterization of odd, monotone, unbiased voting rules:

Lemma 3. Let $\Phi:\{-1,1\}^{n} \rightarrow\{-1,1\}$ be an odd, monotone voting rule with minimal winning coalitions $\mathcal{F} . \Phi$ is unbiased if and only if every voter $i$ is pivotal for an equal number of winning coalitions of each size.

Proof. Let $\mathcal{F}_{i}=\{T: \exists ! S \in \mathcal{F}$ s.t. $i \in S$ and $S \subseteq T\}$ be the set of winning coalitions of $\Phi$ for which voter $i$ is pivotal, and let $\mathcal{F}_{i, j}=\left\{S \in \mathcal{F}_{i}:|S|=j\right\}$ be the subset of these coalitions with size $j$. If we let $m_{i, j}=\left|\mathcal{F}_{i, j}\right|$, then for any probability measure $p$ on $\{-1,1\}$, voter $i$ has influence

$$
\begin{aligned}
\mathbb{I}_{i}^{p^{\otimes n}}(\Phi)= & \sum_{S \in \mathcal{F}_{i}} \underset{x \sim p^{\otimes n}}{\mathbb{P}}\left[\left(x_{j}=y \forall j \in S \backslash\{i\}\right) \wedge\left(x_{j}=-y \forall j \notin S \backslash\{i\}\right)\right] \\
& +\sum_{S \in \mathcal{F}_{i}} \underset{x \sim p^{\otimes n}}{\mathbb{P}}\left[\left(x_{j}=y \forall j \in S\right) \wedge\left(x_{j}=-y \forall j \notin S\right)\right] \\
= & \sum_{j=1}^{n} m_{i, j} c_{j}(p)
\end{aligned}
$$

where, with the slight abuse of notation $\mathbb{P}_{x \sim p}[x=1]=p$, the coefficients are $c_{j}(p)=$ $p^{j-1}(1-p)^{n-j+1}+p^{n-j+1}(1-p)^{j-1}+p^{j}(1-p)^{n-j}+p^{n-j}(1-p)^{j}$. Because $\Phi$ is unbiased, we know that $\sum_{j=1}^{n} m_{1, j} c_{j}(p)=\cdots=\sum_{j=1}^{n} m_{n, j} c_{j}(p)$ for all $p$. This is possible if and only if $m_{1, j}=\cdots=m_{n, j}$ for each $j \in[n]$.

We can also lower bound the size of the smallest winning coalition of any odd, monotone, unbiased voting rule:

Lemma 4. Let $\Phi:\{-1,1\}^{n} \rightarrow\{-1,1\}$ be an odd, monotone, unbiased voting rule with minimal winning coalitions $\mathcal{F}$. Then, $|S| \geqslant\lceil\sqrt{n}\rceil$ for all $S \in \mathcal{F}$.

Proof. Let $k=\min \{|S|: S \in \mathcal{F}\}$ be the size of a smallest winning coalition of $\Phi$ (call it a minimum winning coalition) and let $\mathcal{F}^{*}=\bigcup_{i=1}^{n} \mathcal{F}_{i}^{*}$ be the set of all minimum winning coalitions. Let $m_{i}=\left|\left\{S \in \mathcal{F}^{*}: i \in S\right\}\right|$ and $m=\left|\mathcal{F}^{*}\right|$. Since $\Phi$ is unbiased and every participating voter is pivotal for a minimum winning coalition, $m_{1}=\cdots=m_{n}=m k / n$ by Lemma 3 and the fact that $\sum_{i=1}^{n} m_{i}=m k$.

Therefore, for any $S \in \mathcal{F}^{*}, \sum_{i \in S} \sum_{T \in \mathcal{F}} \mathbb{1}[i \in T]=\sum_{i \in S} m_{i}=m k^{2} / n$. This implies that the elements of $S$ collectively appear in at most $m k^{2} / n$ different $T \in \mathcal{F}^{*}$. Finally, because $\mathcal{F}^{*} \subseteq \mathcal{F}$ is an intersecting family of sets with $\left|\mathcal{F}^{*}\right|=m$, we can conclude that $m \leqslant m k^{2} / n \Longrightarrow k \geqslant \sqrt{n}$. Since $k \in \mathbb{Z}$, this implies that $k \geqslant\lceil\sqrt{n}\rceil$.

It is worth noting that [2] proved that $|S| \geqslant\lceil\sqrt{n}\rceil$ for all $S \in \mathcal{F}$ when $\Phi$ is transitivesymmetric (Theorem 2), and moreover, there exist transitive-symmetric $\Phi$ for which this 
lower bound is tight (Theorem 6). Our Lemma 4 has just shown that the lower bound even holds under the weaker condition $\Phi$ is unbiased. Our Theorem 16 proves that this lower bound is tight up to a constant factor if $\Phi$ is unbiased but not transitive-symmetric, but it is unclear whether the bound is achieved exactly by any voting rules that are unbiased but not transitive-symmetric.

\subsection{Graphic Voting Rules}

We consider voting rules that equate the set of voters with the vertices of an underlying graph $G$. To avoid treating ties in a voting model that only admits strict preferences, we assume $|V|=n$ is odd. The graphic voting rule associated to a graph $G$ of radius $r$ is just $\Phi_{G}=\Phi_{B_{r}(G)}$, i.e.

$$
\Phi_{G}(x)=\Phi_{B_{r}(G)}(x)= \begin{cases}y & \exists v \in V \text { s.t. } x_{i}=y \forall i \in B_{r}(v) \\ \operatorname{maj}_{n}(x) & \text { otherwise }\end{cases}
$$

We make a few remarks about this definition, some of which will motivate us to impose additional assumptions on the graph $G$ underlying the voting rule $\Phi_{G}$.

1. $\Phi_{G}$ is a valid voting rule that is odd and monotone because $B_{r}(G)$ is an intersecting family of sets by our definition of the graph radius.

2. Because $\Phi_{G}$ is uniquely determined by $G$ 's balls of radius $r$, we know that aut $\left(\Phi_{G}\right) \subseteq$ $\operatorname{aut}\left(B_{r}(G)\right)$. So if $B_{r}(G)$ is not vertex-transitive, then $\Phi_{G}$ is not transitive-symmetric.

3. We can assume that $\operatorname{diam}(G)=2$ (and therefore $\operatorname{rad}(G)=1$ ) without loss of generality: if $\operatorname{rad}(G)=r$, let $G^{\prime}=\left(V,\left\{\{u, v\}: u \in V, v \in B_{r}(u)\right\}\right)$ be the graph obtained by connecting all the vertices of $G$ in balls of radius $r$. It is easy to see that $\operatorname{rad}\left(G^{\prime}\right)=1$ and $\Phi_{G}=\Phi_{G^{\prime}}$. We ignore the trivial case where where $\operatorname{diam}\left(G^{\prime}\right)=1$ and $G^{\prime}$ is just the complete graph.

4. We further assume that $G$ is $d$-regular and has all balls of radius 1 distinct. Then, every voter in $\Phi_{G}$ participates in exactly $d+1$ minimum winning coalitions, each of size $d+1$.

5. To ensure that $\Phi_{G} \neq \mathrm{maj}_{n}$, we further assume that $G$ is $d$-regular with $d \leqslant(n-3) / 2$.

Theorem 6 shows that 3 - 5 are reasonable assumptions, even in conjunction. We now provide an explicit example of an odd, monotone voting rule on $n=11$ voters that is unbiased but not transitive-symmetric:

Theorem 5. Let $G$ be the graph depicted in Figure 1b. Then $\Phi_{G}$ is an odd, monotone, unbiased voting rule with $\left|\operatorname{aut}\left(\Phi_{G}\right)\right|=1$, i.e. $\Phi_{G}$ is totally asymmetric.

Proof. $G$ is a 4-regular graph on 11 vertices with $\operatorname{diam}(G)=2$ and all balls of radius 1 distinct. One can also check through exhaustive computation that aut $\left(B_{1}(G)\right)$ is trivial. 
Therefore, $\Phi_{G}:\{-1,1\}^{11} \rightarrow\{-1,1\}$ is such that aut $\left(\Phi_{G}\right) \subseteq \operatorname{aut}\left(B_{1}(G)\right)=\{\operatorname{id}\}$, and each of the 11 voters participates in exactly 5 minimum winning coalitions of size 5 . Because these are the only winning coalitions in which $\Phi_{G}$ differs from majority rule, every voter is pivotal to an equal number of winning coalitions of each size. By Lemma 3, this implies that $\Phi_{G}$ is unbiased.

\section{A Probabilistic Construction}

Here, we use the probabilistic method to prove the existence of a class of regular graphs whose associated graphic voting rules are totally asymmetric, unbiased, and have winning coalitions of size $\lfloor 0.293 n\rfloor$. This result immediately implies our main Theorem 1 . More specifically, let $\mathcal{G}_{n, d}$ be the uniform distribution over $d$-regular graphs on $n$ vertices, where $n d$ is even. For our purposes, this means that $n$ is odd and $d$ is even. Theorem 6 shows that probability of sampling a graph of interest from $\mathcal{G}_{n, d}$ is $1-o(1)$, as long as the degree $d$ is large enough:

Theorem 6. Let $G \sim \mathcal{G}_{n, d}$ with $\sqrt{n} \log n \leqslant d \leqslant n / 2$. Then, with probability $1-o(1)$, $\left|\operatorname{aut}\left(B_{1}(G)\right)\right|=1$ and $\left|B_{1}(u) \cap B_{1}(v)\right|=(1+o(1)) d^{2} / n$ for every pair of vertices $u \neq v$.

When taken together with the following result, our main Theorem 1 follows:

Theorem 7. Let $G$ be a d-regular graph with $\lfloor 0.293 n\rfloor-1 \leqslant d \leqslant(n-3) / 2$, $\left|\operatorname{aut}\left(B_{1}(G)\right)\right|=$ 1 , and $\left|B_{1}(u) \cap B_{1}(v)\right|=(1+o(1)) d^{2} / n$ for every pair of vertices $u \neq v$. If $n$ is odd, then $\Phi_{G}$ is an odd, monotone, unbiased voting rule with $\left|\operatorname{aut}\left(\Phi_{G}\right)\right|=1$. Moreover, $\Phi_{G}$ has winning coalitions of size $d+1$.

Proof. Because $0<\left|B_{1}(u) \cap B_{1}(v)\right|=(1+o(1)) d^{2} / n<d+1$ for all $u \neq v \in V$, we know that $\operatorname{rad}(G)=1$ and all balls of radius 1 are distinct. Therefore, $\operatorname{aut}\left(\Phi_{G}\right) \subseteq \operatorname{aut}\left(B_{1}(G)\right)=$ $\{$ id $\}$, and each voter participates in exactly $d+1$ minimum winning coalitions of size $d+1$. Now, we prove that $\Phi_{G}$ is unbiased.

Let $k=\max _{u \neq v}\left|B_{1}(u) \cap B_{1}(v)\right|=(1+o(1)) d^{2} / n$ be the maximal intersection between any two minimum winning coalitions of $\Phi_{G}$, and consider the following families of winning coalitions for each $v \in V$ :

$$
\mathcal{F}_{v}=\left\{S \supseteq B_{1}(v):|S|<\min \{2(d+1)-k,(n+1) / 2\}\right\}
$$

Because $|S|<2(d+1)-k \leqslant\left|B_{1}(u) \cup B_{1}(v)\right|$ for all $S \in \mathcal{F}$ and $u \neq v \in V$, we know that $\mathcal{F}_{u} \cap \mathcal{F}_{v}=\varnothing$ and $\left|\left\{S \in \mathcal{F}_{u}:|S|=t\right\}\right|=\left|\left\{S \in \mathcal{F}_{v}:|S|=t\right\}\right|$ for all $t$. Moreover, if $S \in \mathcal{F}_{v}$, then $S \backslash\{u\}$ is not a winning coalition for any $u \in B_{1}(v)$ (since no $S \in \mathcal{F}_{v}$ is a majority). In words, every voter in $B_{1}(v)$ is pivotal to every winning coalition in $\mathcal{F}_{v}$.

Now assume that $2(d+1)-k \geqslant(n+1) / 2$. Then, $\bigcup_{v \in V} \mathcal{F}_{v}$ is exactly the set of all winning coalitions smaller than a majority. Because every voter $v \in V$ participates in exactly $d+1$ minimum winning coalitions $B_{1}(u)$ of size $d+1$, the analysis above implies that every voter is pivotal for an equal number of winning coalitions of each size. By Lemma 3 , this implies that $\Phi_{G}$ is unbiased. 
Finally, we translate the assumption that $2(d+1)-k \geqslant(n+1) / 2$ into an assumption about $d$. Since, $k=(1+o(1)) d^{2} / n$, we solve the quadratic inequality $2(d+1)-(1+$ $o(1)) d^{2} / n \geqslant(n+1) / 2$ to obtain the condition that $d \geqslant(1-1 / \sqrt{2}+o(1)) n$. For $n$ large enough, $d \geqslant\lfloor 0.293 n\rfloor-1$ will suffice.

The remainder of this section proves Theorem 6 as a concentration inequality about the defect of a random regular graph. Intuitively, the defect of a graph $G$ quantifies just how asymmetric its balls $B_{1}(G)$ of radius 1 are. More formally, we have the following definition and lemma, inspired by [4]:

Definition 8. Let $G=(V, E)$ be a graph, and let $\sigma, \pi \in S_{n}$ be permutations of $V$. The defect of a vertex $v$ with respect to $\sigma$ and $\pi$ is

$$
D_{\sigma, \pi}(v)=\left|\sigma\left(B_{1}(v)\right) \Delta B_{1}(\pi(v))\right|
$$

where $\Delta$ denotes the symmetric difference. Analogously, we define the defect of the graph $G$ with respect to $\sigma$ and $\pi$ as $D_{\sigma, \pi}(G)=\max _{v \in V} D_{\sigma, \pi}(v)$.

Lemma 9. Let $G=(V, E)$ be a graph and $\sigma \in S_{n}$ a permutation of $V$. Then, $\sigma \in$ $\operatorname{aut}\left(B_{1}(G)\right)$ if and only if there exists another permutation $\pi \in S_{n}$ such that $D_{\sigma, \pi}(G)=0$. Moreover, $\sigma \in \operatorname{aut}(G)$ if and only if $D_{\sigma, \sigma}(G)=0$.

Proof. By definition, $\sigma$ is an automorphism of $B_{1}(G)$ if and only if for every vertex $v \in V$, there is another vertex $v^{\prime} \in V$ such that $\sigma\left(B_{1}(v)\right)=B_{1}\left(v^{\prime}\right)$. In the case where all balls $B_{1}(v)$ are distinct, this is possible if and only if the mapping $v \mapsto v^{\prime}$ is a permutation, which we will call $\pi$. In the general case, $\pi$ need not be bijective, but there will always be a bijective $\pi$ for which the desired statement holds biconditionally. Since $\sigma\left(B_{1}(v)\right)=$ $B_{1}(\pi(v))$ for all $v \in V, D_{\sigma, \pi}(G)=0$. The second statement follows from the fact that $\sigma \in \operatorname{aut}(G)$ if and only if $\{\sigma(u), \sigma(v)\} \in E$ for every edge $\{u, v\} \in E$. This is equivalent to saying that $B_{1}(\sigma(v))=\sigma\left(B_{1}(v)\right)$ for every vertex $v \in V$.

Our proof of Theorem 6 closely follows the approach of [4]. Subsection 3.1 proves a concentration inequality about the defect of an Erdös-Rényi random graph, and Subsection 3.2 uses that result to prove an analogous statement (from which Theorem 6 follows) about random regular graphs.

\subsection{A Result About Erdős-Rényi Random Graphs}

The Erdős-Rényi random graph distribution $G(n, p)$ is the probability space over graphs with vertex set $V=[n]$, where each of the $\left(\begin{array}{l}n \\ 2\end{array}\right)$ possible edges is present independently with probability $p$. In this subsection, we prove the following concentration inequality about the defect of a graph drawn from the Erdős-Rényi distribution $G(n, p)$. The proof closely follows that of Theorem 3.1 in [4]: 
Theorem 10. Let $G \sim G(n, p)$ with $p=\omega(\log n / n)$ and $1-p=\omega(\log n / n)$. Then, there exist permutations $\sigma, \pi \in S_{n}$ such that $\mid\{v \in V: \sigma(v) \neq v$ or $\pi(v) \neq v\} \mid \geqslant \ell$ and $D_{\sigma, \pi}(G)<(2-o(1)) n p(1-p)$ with probability at most

$$
4 \sum_{k=\ell}^{n} \exp \left(-c \epsilon^{2} n p(1-p)+2 \log n\right)^{k}=o(1)
$$

for some constant $c>0$ and any $\epsilon=\epsilon(n)$ such that $\epsilon=o(1)$ and $\epsilon^{2} n p(1-p)=\omega(\log n)$. For example, $p=\log (n)^{2} / n$ and $\epsilon=\log (n)^{-1 / 3}$ suffice.

Intuitively, Theorem 10 implies that $B_{1}(G)$ from almost every $G \sim G(n, p)$ is highly asymmetric. We state the probability bound in a particularly technical way to facilitate the proof of an analogous theorem about random $n p$-regular graphs in Subsection 3.2. The centerpiece of our proof of Theorem 10 is the following concentration inequality, which represents a refinement of McDiarmid's bounded difference inequality [6] to the special case of Bernoulli random variables:

Lemma 11 (Alon et al. [1]). Let $X$ be a random variable on a probability space generated by finitely many independent Bernoulli random variables $Y_{i} \sim \operatorname{Bern}\left(p_{i}\right)$. Let $\delta$ be such that changing any $Y_{i}$ (keeping all others the same) can change $X$ by at most $\delta$. Define $\Delta^{2}=\delta^{2} \sum_{i} p_{i}\left(1-p_{i}\right)$. Then, for all $0<t<2 \Delta / \delta, \mathbb{P}[|X-\mathbb{E}[X]|>t \Delta] \leqslant 2 e^{-t^{2} / 4}$.

Proof (Theorem 10). Let $\sigma, \pi \in S_{n}$ be permutations of the vertices of $G$, and let $U=$ $\{v \in V: \sigma(v) \neq v$ or $\pi(v) \neq v\}$ be the vertices not fixed in place by $\sigma$ and $\pi$. Let $k=|U|$, and assume that $k>0$ for non-triviality. Next, let $X=\sum_{v \in U} D_{\sigma, \pi}(v)$. We will apply Lemma 11 to the random variable $X$ later in the proof.

For any vertex $v \in U$, let $E_{1}^{u v}$ be the event that $u \in \sigma\left(B_{1}(v)\right) \backslash B_{1}(\pi(v))$ and let $E_{2}^{u v}$ be the event that $u \in B_{1}(\pi(v)) \backslash \sigma\left(B_{1}(v)\right)$. $E_{1}^{u v}$ occurs if and only if $\left\{\sigma^{-1}(u), v\right\} \in E$ and $\{u, \pi(v)\} \notin E$, while $E_{2}^{u v}$ occurs if and only if $\{u, \pi(v)\} \in E$ and $\left\{\sigma^{-1}(u), v\right\} \notin E$. Since $v$ is moved by at least one of $\sigma$ or $\pi$ (by the assumption that $v \in U$ ), this implies that

$$
\mathbb{E}\left[D_{\sigma, \pi}(v)\right]=\sum_{u \in V} \mathbb{P}\left[E_{1}^{u v} \cup E_{2}^{u v}\right]= \begin{cases}2(n-1) p(1-p) & \pi(\sigma(v))=v \\ 2(n-2) p(1-p) & \text { otherwise }\end{cases}
$$

where the terms $(n-1)$ and $(n-2)$ account for cases where $u=\pi(v)$ and $\sigma^{-1}(u)=v$. Therefore,

$$
\mathbb{E}[X]=\sum_{v \in U} \mathbb{E}\left[D_{\sigma, \pi}(v)\right]=(2-o(1)) k n p(1-p)
$$

Now, we analyze how the presence of edges in $G$ influences the value of $X$. First, we note that $X$ only depends on edges of the graph incident to vertices in $U$. There are $k(n-k)+\left(\begin{array}{l}k \\ 2\end{array}\right)$ such edges. Second, adding or deleting an edge $\{u, v\}$ from $G$ can only change the values of the following six summands, each by at most 1: $D_{\sigma, \pi}(u), D_{\sigma, \pi}(v)$, $D_{\sigma, \pi}\left(\sigma^{-1}(u)\right), D_{\sigma, \pi}\left(\sigma^{-1}(v)\right), D_{\sigma, \pi}\left(\pi^{-1}(u)\right)$, and $D_{\sigma, \pi}\left(\pi^{-1}(v)\right)$. This means that $X$ satisfies 
the hypothesis of Lemma 11 with parameters $\delta=6$ and $\Delta^{2}=36\left(k(n-k)+\left(\begin{array}{l}k \\ 2\end{array}\right)\right) p(1-p)=$ $\Theta(k n p(1-p))$. So for some constant $c>0$,

$$
\mathbb{P}[|X-\mathbb{E}[X]|>2 \epsilon k n p(1-p)] \leqslant \exp \left(-c \epsilon^{2} k n p(1-p)\right)
$$

Therefore, with probability at least $1-\exp \left(-c \epsilon^{2} k n p(1-p)\right)$,

$$
D_{\sigma, \pi}(G) \geqslant \max _{v \in U} D_{\sigma, \pi}(v) \geqslant \frac{1}{k}(\mathbb{E}[X]-2 \epsilon k n p(1-p))=(2-o(1)) n p(1-p)
$$

To bound the probability that this bound holds for all $\sigma, \pi \in S_{n}$ for which $k=|U| \geqslant \ell$, we will take a union bound. There are at most $\left(\begin{array}{l}n \\ k\end{array}\right) k ! \leqslant n^{k}$ permutations that exactly fix $n-k$ vertices, so there are at most $\sum_{i=0}^{k} \sum_{j=0}^{k} n^{i} n^{j} \leqslant 4 n^{2 k}$ pairs of permutations $\pi$ and $\sigma$ that fix exactly $n-k$ vertices between them, i.e. for which $|U|=k$. Therefore, there exists a nontrivial pair of permutations $\pi$ and $\sigma$ for which $|U| \geqslant \ell$ and $D_{\sigma, \pi}(G)<(2-o(1)) n p(1-p)$ with probability at most

$$
\sum_{k=\ell}^{n} 4 n^{2 k} \exp \left(-c \epsilon^{2} k n p(1-p)\right)=4 \sum_{k=\ell}^{n} \exp \left(-c \epsilon^{2} n p(1-p)+2 \log n\right)^{k}
$$

\subsection{Proof of Theorem 6}

Theorem 6 follows immediately from Lemmas 12 and 13:

Lemma 12 (Krivelevich et al. [5]). Let $G \sim \mathcal{G}_{n, d}$. If $\sqrt{n} \log n \leqslant d \leqslant n-n / \log _{2} n$, then with probability $1-o(1),\left|B_{1}(u) \cap B_{1}(v)\right|=(1+o(1)) d^{2} / n$ for every pair of vertices $u \neq v$ in $G$. If instead $\log n \leqslant d \leqslant \sqrt{n} \log n$, then with probability $1-o(1)$, there exists a constant $\epsilon>0$ such that $\left|B_{1}(u) \cap B_{1}(v)\right|<d^{1-\epsilon}$ for every pair of vertices $u \neq v$ in $G$.

Lemma 13. Let $G \sim \mathcal{G}_{n, d}$, with $d=\omega(\log n)$ and $d \leqslant n / 2$. With probability $1-o(1)$, $\left|\operatorname{aut}\left(B_{1}(G)\right)\right|=1$, i.e. $B_{1}(G)$ is totally asymmetric.

To prove Lemma 13, we will need two additional technical lemmas from [4]:

Lemma 14 (Kim et al. [4]). Let $G \sim \mathcal{G}_{n, d}$ with $d \leqslant n^{3 / 4}$ and $d=\omega(\log n)$. Then, with probability $1-o(1)$, every subset of vertices in $G$ of size $a \leqslant n d^{-1 / 3}$ spans at most $d a / \log d$ edges.

Lemma 15 (Kim et al. [4]). Let $G \sim G(n, d / n)$. For any constant $\delta>0$ and $n$ sufficiently large, $G$ is d-regular with probability at least $\exp \left(-n d^{1 / 2+\delta}\right)$.

Proof (Lemma 13). The proof closely follows Section 5 of [4], which proves an analogous result for aut $(G)$, rather than aut $\left(B_{1}(G)\right)$. Our strategy for adapting their proof is similar to the way we proved Theorem 10. Re-introducing notation from that proof, for any nontrivial pair permutations $\sigma, \pi \in S_{n}$, we will let $U=\{v \in V: \pi(v) \neq v$ or $\sigma(v) \neq v\}$ and 
$W=V \backslash U$. The following observation is critical:

$$
\begin{aligned}
D_{\sigma, \pi}(v) \geqslant & \left|\left(\sigma\left(B_{1}(v)\right) \cap W\right) \Delta\left(B_{1}(\pi(v)) \cap W\right)\right| \\
= & \left(d+1-\left|\sigma\left(B_{1}(v)\right) \cap U\right|\right)+\left(d+1-\left|B_{1}(\pi(v)) \cap U\right|\right) \\
& -2\left|\sigma\left(B_{1}(v)\right) \cap B_{1}(\pi(v)) \cap W\right| \\
= & 2 d+2-\left|B_{1}(v) \cap U\right|-\left|B_{1}(\pi(v)) \cap U\right|-2\left|B_{1}(v) \cap B_{1}(\pi(v)) \cap W\right| \\
\geqslant & 2 d-\left|B_{1}(v) \cap U\right|-\left|B_{1}(\pi(v)) \cap U\right|-2\left|B_{1}(v) \cap B_{1}(\pi(v))\right|
\end{aligned}
$$

Inequality 1 follows because that $\sigma(v) \in U$ for all $v \in U$ and $\sigma(v)=v$ for all $v \in V \backslash U$. Now, we enumerate cases.

1. $n^{3 / 4} \leqslant d \leqslant n / 2$. Then, Lemma 12 tells us that $\left|B_{1}(v) \cap B_{1}(\pi(v))\right|=(1+o(1)) d^{2} / n$ with probability $1-o(1)$. If we let $\ell=d^{1-\delta}=o(d)$ for some small constant $\delta>0$ $(\delta=1 / 100$ suffices $)$, we need to analyze two additional sub-cases:

(a) $|U|>\ell$. For any graph $G$, let $B_{G}$ denote the event that there exist permutations $\sigma, \pi \in S_{n}$ such that $|U|>\ell$ and $D_{\sigma, \pi}\left(G^{\prime}\right)<(2-o(1)) d(1-d / n)$. By Theorem 10

$$
\underset{G^{\prime} \sim G(n, d / n)}{\mathbb{P}}\left[B_{G^{\prime}}\right] \leqslant 4 \sum_{k=\ell+1}^{n} \exp \left(-c \epsilon^{2} d(1-d / n)+2 \log n\right)^{k}
$$

for an appropriately chosen constant $c$. If we constrain $\epsilon \geqslant d^{-\delta}$, our assumption that $\ell=d^{1-\delta}$ tells us that this probability is at most $\exp \left(-\Omega\left(d^{2-3 \delta}\right)\right)$ and therefore

$$
\begin{aligned}
\underset{G \sim \mathcal{G}_{n, d}}{\mathbb{P}}\left[B_{G}\right] & =\underset{G^{\prime} \sim G(n, d / n)}{\mathbb{P}}\left[B_{G^{\prime}} \mid G^{\prime} d \text {-regular }\right] \\
& \leqslant \underset{G^{\prime} \sim G(n, d / n)}{\mathbb{P}}\left[B_{G^{\prime}}\right] / \underset{G^{\prime} \sim G(n, d / n)}{\mathbb{P}}\left[G^{\prime} \text { d-regular }\right] \\
& \leqslant \exp \left(-\Omega\left(d^{2-3 \delta}\right)+n d^{1 / 2+\delta}\right)
\end{aligned}
$$

The second-to-last line uses the fact that $\mathbb{P}[A \mid B] \leqslant \mathbb{P}[A] / \mathbb{P}[B]$, and the last line invokes Lemma 15 , which says that $G^{\prime}$ is regular with probability at least $\exp \left(-n d^{1 / 2+\delta}\right)$. Finally, because we constrain $n^{3 / 4} \leqslant d \leqslant n / 2$, the final quantity is $o(1)$ as desired.

(b) $|U| \leqslant \ell$. Then, $\left|B_{1}(v) \cap U\right| \leqslant|U|=o(d)$ and $\left|B_{1}(\pi(v)) \cap U\right| \leqslant|U|=o(d)$. Substituting these quantities into Inequality 2 , we get that with probability $1-o(1), D_{\sigma, \pi}(v) \geqslant(2-o(1)) d(1-d / n)$ for all non-trivial pairs of permutations $\sigma$ and $\pi$ for which $|U| \leqslant \ell$.

2. $d \leqslant n^{3 / 4}$ and $d=\omega(\log n)$. Then, by Lemma $12,\left|B_{1}(v) \cap B_{1}(\pi(v))\right|=o(d)$ for all vertices $v$ with probability $1-o(1)$. If we let $\ell=n d^{-1 / 3} \geqslant d$, we have two-sub-cases again: 
(a) $|U|>\ell$. The analysis is nearly identical to case $1(\mathrm{a})$, and proves the same result.

(b) $|U| \leqslant \ell$. By Lemma 14, with probability $1-o(1)$, every subset $U \subseteq V$ of vertices with $|U| \leqslant \ell$ spans at most $d|U| / \log d=o(d)$ edges. This implies that with probability $1-o(1)$, every such $U$ contains a vertex $v \in U$ such that $\left|B_{1}(v) \cap U\right|=o(d)$ and $\left|B_{1}(\pi(v)) \cap U\right|=o(d)$. So substituting into Inequality 2 , we get that with probability $1-o(1), D_{\sigma, \pi}(G) \geqslant(2-o(1)) d \geqslant(2-o(1)) d(1-$ $d / n)$ for every non-trivial pair of permutations $\sigma$ and $\pi$ for which $|U| \leqslant \ell$.

Therefore, we can conclude that with probability $1-o(1), D_{\sigma, \pi}(G) \geqslant(2-o(1)) d(1-$ $d / n)$ for every non-trivial pair permutations $\sigma$ and $\pi$. By Lemma 9, this result implies that $\left|\operatorname{aut}\left(B_{1}(G)\right)\right|=1$ with probability $1-o(1)$.

\section{An Explicit Construction}

In this section, we explicitly construct a voting rule that is unbiased, neither transitivesymmetric nor asymmetric, and has winning coalitions of size $O(\sqrt{n})$ :

Theorem 16. Let $m=q^{2}+q+1$ for some prime power $q$, and let $n=11 m$. Then, there exists an odd, monotone, unbiased voting rule $\Phi:\{-1,1\}^{n} \rightarrow\{-1,1\}$ that is not transitive symmetric and has winning coalitions of size $\lfloor 1.508 \sqrt{n}\rfloor+5$.

We prove Theorem 16 by using the procedure we describe in Lemma 17 to compose the unbiased but asymmetric graphic voting rule $\Phi_{1}$ of Theorem 5 with a transitive-symmetric voting rule $\Phi_{2}$ described by [2]. Informally, we consider $n_{2}$ groups of $n_{1}$ voters each. We let each of these groups make a decision using $\Phi_{1}$ and then aggregate these group decisions using $\Phi_{2}$. More formally,

Lemma 17. Let $\Phi_{1}:\{-1,1\}^{n_{1}} \rightarrow\{-1,1\}$ be an odd, monotone, unbiased voting rule that is not transitive-symmetric and has winning coalitions of size $d_{1}$. Let $\Phi_{2}:\{-1,1\}^{n_{2}} \rightarrow$ $\{-1,1\}$ be an odd, monotone, transitive-symmetric voting rule that has winning coalitions of size $d_{2}$. Then, there exists a voting rule $\Phi:\{-1,1\}^{n_{1} \times n_{2}} \rightarrow\{-1,1\}$ that has winning coalitions of size $d_{1} d_{2}$, and is odd, monotone, unbiased, and not transitive-symmetric.

Proof. For $X \in\{-1,1\}^{n_{1} \times n_{2}}$, let $X^{(i)}=\left(x_{1, i}, \ldots, x_{n_{1}, i}\right)^{T} \in\{-1,1\}^{n_{2}}$ be the $i^{\text {th }}$ column vector of $X$, and define

$$
\Phi(X)=\Phi_{2}\left(\Phi_{1}\left(X^{(1)}\right), \ldots, \Phi_{1}\left(X^{\left(n_{2}\right)}\right)\right)
$$

$\Phi$ is odd and monotone because $\Phi_{1}$ and $\Phi_{2}$ are also odd and monotone. We can form a winning coalition of size $d_{1} d_{2}$ for $\Phi$ by taking a winning coalition for $\Phi_{2}$ and assigning to each member of that coalition an entire winning coalition for $\Phi_{1}$.

Now, we show that $\Phi$ is unbiased. Because $\Phi_{1}$ is unbiased, every $x_{i, j}$ has an equal probability of being pivotal to $\Phi_{1}\left(X^{(j)}\right)$. Because $\Phi_{2}$ is unbiased, every $\Phi_{1}\left(X^{(j)}\right)$ has an 
equal probability of being pivotal to $\Phi(X)=\Phi_{2}\left(\Phi_{1}\left(X^{(1)}\right), \ldots, \Phi_{1}\left(X^{\left(n_{2}\right)}\right)\right)$. So every $x_{i, j}$ has an equal probability of being pivotal to $\Phi(X)$, i.e. $\Phi$ is unbiased.

Finally, we show that $\Phi$ is not transitive-symmetric. Let $\sigma \in \operatorname{aut}(\Phi)$, so $\Phi(X)=$ $\Phi\left(\sigma^{-1}(X)\right)$ for all $X \in\{-1,1\}^{n_{1} \times n_{2}}$. By the definition of $\Phi$ and the fact that $\Phi_{2}$ is non-constant, each $X$ must have some $\sigma_{2, X} \in \operatorname{aut}\left(\Phi_{2}\right)$ such that

$$
\left(\Phi_{1}\left(\sigma^{-1}(X)^{(1)}\right), \ldots, \Phi_{1}\left(\sigma^{-1}(X)^{\left(n_{2}\right)}\right)\right)=\left(\Phi_{1}\left(X^{\sigma_{2, X}^{-1}(1)}\right), \ldots, \Phi_{1}\left(X^{\sigma_{2, X}^{-1}\left(n_{2}\right)}\right)\right)
$$

Because this must hold for all $X$ and $\Phi_{1}$ is also non-constant, this further implies that each $X$ must also have some $\sigma_{1, X} \in \operatorname{aut}\left(\Phi_{1}\right)$ such that

$$
\sigma^{-1}(X)^{(i)}=\sigma_{1, X}^{-1}\left(X^{\left(\sigma_{2, X}^{-1}(i)\right)}\right)
$$

for all $i \in\left[n_{2}\right]$. However, because $\Phi_{1}$ is not transitive-symmetric, there must be some $u, v \in\left[n_{1}\right]$ such that $\sigma_{1, X}(u) \neq v$ for all $\sigma_{1, X} \in \operatorname{aut}\left(\Phi_{1}\right)$. Therefore, we can conclude that $\Phi$ is not transitive-symmetric.

Proof (Theorem 16). Theorem 5 describes a voting rule $\Phi_{1}:\{-1,1\}^{11} \rightarrow\{-1,1\}$ that is odd, monotone, unbiased, not transitive-symmetric, and has winning coalitions of size 5 . Theorem 6 of [2] constructs a voting rule $\Phi_{2}:\{-1,1\}^{m} \rightarrow\{-1,1\}$ that is odd, monotone, transitive-symmetric, and has winning coalitions of size $\lceil\sqrt{m}$. Lemma 17 provides an algorithm to compose $\Phi_{1}$ and $\Phi_{2}$ to obtain a voting rule $\Phi:\{-1,1\}^{11 m} \rightarrow\{-1,1\}$ that is odd, monotone, unbiased, not transitive-symmetric, and has winning coalitions of size $5\lceil\sqrt{m}\rceil \frac{5 \sqrt{n}}{\sqrt{11}}+5 \leqslant\lfloor 1.508 \sqrt{n}\rfloor+5$.

\section{Conclusions and Future Work}

We have provided two proofs that there exist voting rules which are unbiased but not transitive-symmetric. At the crux of our arguments is a class of voting rules defined by $d$-regular graphs of diameter 2 .

Section 3 provides a probabilistic proof that there exist voting rules that are unbiased and totally asymmetric (not just not transitive-symmetric). Theorem 6 shows that with probability $1-o(1)$, the voting rules defined by random $d$-regular graphs for $d \geqslant \sqrt{n} \log n$ are totally asymmetric and have all voters participate in exactly $d+1$ minimum winning coalitions of size $d+1$. Theorem 7 shows that if we relax the lower bound on the degree to $d \geqslant 0.293 n-1$, these voting rules are also unbiased with probability $1-o(1)$.

Section 4 explicitly constructs a class of voting rule that is unbiased, but neither transitive-symmetric nor totally asymmetric. On the other hand, this class of voting rules has much smaller winning coalitions of size $O(\sqrt{n})$, which differs by only a constant factor from the lower bound of $\lceil\sqrt{n}\rceil$ that we proved in Lemma 4.

We see two possible directions for future work. First, we conjecture that there exist voting rules that are unbiased, totally asymmetric, and have winning coalitions of size $\sqrt{n} \log n+1$ (i.e. the lower bound of Theorem 7 can be made to match that of Theorem 6). A more sophisticated proof of Lemma 12 may be a fruitful avenue to such a result. 
Second, given the existence of voting rules that are unbiased, neither transitivesymmetric nor totally asymmetric, and have winning coalitions of size $O(\sqrt{n})$, we conjecture that Theorems 6 and 7 can be refined to prove that there exist $d$-regular graphs whose associated voting rules are unbiased but not transitive-symmetric (rather than totally asymmetric) as long as $d \geqslant\lceil\sqrt{n}\rceil-1$ (rather than $d \geqslant \sqrt{n} \log n$ ). In other words, we believe that the lower bound of Lemma 4 is tight not only in general, but also for our construction using graphic voting rules.

\section{Acknowledgments}

I would like to thank Omer Tamuz for pointing me to some useful references and for his helpful feedback on drafts of this paper. I would like to thank Leonard Schulman for his assistance in verifying the correctness of some of the proofs in Section 3.

\section{References}

[1] Noga Alon, Jeong-Han Kim, and Joel Spencer, Nearly perfect matchings in regular simple hypergraphs, Israel Journal of Mathematics 100 (1997), 171-187.

[2] Laurent Bartholdi, Wade Hann-Caruthers, Maya Josyula, Omer Tamuz, and Leeat Yariv, Equitable voting rules, Working paper, 2019.

[3] John Isbell, Homogeneous games II, Proceedings of the American Mathematical Society 11 (1960), 159-161.

[4] Jeong Han Kim, Benny Sudakov, and Van Vu, On the asymmetry of random regular graphs and random graphs, Random Structures and Algorithms 21 (2002), 216-224.

[5] Michael Krivelevich, Benny Sudakov, Van Vu, and Nicholas Wormald, Random regular graphs of high degree, Random Structures and Algorithms 18 (2001), 346-363.

[6] Colin McDiarmid, On the method of bounded differences, Surveys in Combinatorics 141 (1989), 148-188. 